\title{
Real-space Manifestations of Bottlenecks in Turbulence Spectra
}

\author{
Uriel Frisch, ${ }^{1}$ Samriddhi Sankar Ray, ${ }^{1}$ Ganapati Sahoo, ${ }^{2}$ Debarghya Banerjee,${ }^{3}$ and Rahul Pandit,$*$ \\ ${ }^{1}$ Laboratoire Lagrange, OCA, UNS, CNRS, BP 4229, 06304 Nice Cedex 4, France \\ ${ }^{2}$ Max Planck Institute for Dynamics and Self-Organization, Am Fassberg 17, 37077 Göttingen, Germany \\ ${ }^{3}$ Centre for Condensed Matter Theory, Department of Physics, Indian Institute of Science, Bangalore, India
}

(Dated: August 10, 2018)

\begin{abstract}
An energy-spectrum bottleneck, a bump in the turbulence spectrum between the inertial and dissipation ranges, is shown to occur in the non-turbulent, one-dimensional, hyperviscous Burgers equation and found to be the Fourier-space signature of oscillations in the real-space velocity, which are explained by boundary-layer-expansion techniques. Pseudospectral simulations are used to show that such oscillations occur in velocity correlation functions in one- and three-dimensional hyperviscous hydrodynamical equations that display genuine turbulence.

PACS numbers: 47.27 Gs, 47.10.ad
\end{abstract}

The energy spectrum $E(k)$ characterizes the statistical distribution of kinetic energy among the wavenumbers $k$ in homogeneous, isotropic, fluid turbulence in three dimensions (3D). If $k_{I}$ and $k_{d}$ denote, respectively, the wave-vector magnitudes related to the inverses of the lengths $L_{I}$, at which energy is injected into the system, and $\eta_{d}$, where viscous dissipation becomes significant, then, in the inertial range $k_{I} \ll k \ll k_{d}$, this spectrum scales as $E(k) \propto k^{-n}$; the phenomenological theory (K41) of Kolmogorov [1], which does not account for intermittency [2], yields $n=5 / 3$ for 3D fluid turbulence. In the far dissipation range $k \gg k_{d}$, this spectrum falls off exponentially (upto algebraic prefactors) [3]. For values of $k$ that lie in between inertial and far-dissipation ranges, a plot of the compensated energy spectrum $(E(k)$ divided by its inertial-range form) versus $k$ exhibits a gentle maximum that is called a bottleneck [4, 5]. Such bottlenecks have been seen in a variety of experiments [,, , 7] and in direct numerical simulations (DNSs) of fluid turbulence [710]. Phenomenological mechanisms have been suggested for the formation of bottlenecks (see, e.g., Refs. [4, 5]). A systematic theoretical study of the bottleneck phenomenon has been initiated in Ref. 11] by using the limit of high dissipativity $\alpha$ in hyperviscous hydrodynamical equations, which have a dissipation operator $\propto\left(-\nabla^{2}\right)^{\alpha}$. Hyperviscous dissipation, with moderate values of $\alpha$, say between 2 and 4, is often used in DNSs in the hope of enhancing the inertial range of scales, but at the price of producing increasingly strong bottlenecks (see Ref. [11] and references therein).

In the first part of our study we develop a quantitative, analytical understanding of bottlenecks, for moderate values of $\alpha$, in the following hyperviscous generalization of the one-dimensional (1D) Burgers equation:

$$
\partial_{t} u+u \partial_{x} u=-\nu_{\alpha} k_{r}^{-2 \alpha}\left(-\partial_{x}^{2}\right)^{\alpha} u+f(x, t) ;
$$

here $u(x, t)$ is the velocity at the point $x$ and time $t$, $\nu_{\alpha}>0$ the hyperviscosity, $k_{r}$ a reference wavenumber, and $f$ the driving force. It is well known that the ordinary $(\alpha=1)$ Burgers equation, with $f=0$, is integrable
[12]; it is also easy to show that its energy spectrum has no bottleneck. By contrast, we show that, for any integer $\alpha>1$, the solution to the hyperviscous Burgers equation (1), in the limit of small $\nu_{\alpha}$, displays an energyspectrum bottleneck; for this it is crucial to examine the solution in real space, where we can use boundary-layertype analysis, in the vicinities of shocks, to uncover oscillations in the velocity profile. We obtain this result both for the unforced, hyperviscous Burgers equation and for its variant (DHB) with deterministic, time-independent, large-scale forcing. We validate our DHB solutions with a pseudospectral DNS. Note that these solutions are timeindependent and not turbulent; however, the key qualitative feature of real-space oscillations in the velocity profile does carry over to oscillations in velocity correlation functions in one- and three-dimensional hyperviscous hydrodynamical equations that display genuine turbulence. We show this in the second part of our study by using DNS. This association of bottlenecks and oscillations in velocity correlation functions has not been made so far. It is akin to the association of peaks in the static structure factor $S(k)$, of a liquid in equilibrium, with damped oscillations in the radial distribution function $g(r)$ [13].

The simplest model presented here, which displays a bottleneck amenable to analytical study, is the DHB equation (11) with integer $\alpha>1$, time-independent force $f=\sin x$, and $u(x, t=0)=0$. The velocity eventually goes to a steady state, which is a solution of the ordinary differential equation (ODE) that is obtained by dropping the time-derivative term in (1). When $\alpha \neq 1$, this nonlinear ODE is not integrable, but its limit as $\nu_{\alpha} \rightarrow 0$ is the same as for ordinary dissipation, namely, it has a shock at $x=\pi$, where the solution jumps from $u_{-}=+2$ to $u_{+}=-2$. For small but finite $\nu_{\alpha}$, the shock is broadened and its structure can be analyzed by a boundary-layer technique using the stretched spatial variable $X \equiv(x-\pi) / \nu^{\beta}$, with $\beta=\frac{1}{2 \alpha-1}$, and expanding the boundary-layer velocity $u^{\mathrm{BL}}$ in powers of $\nu_{\alpha}$ : 
$u^{\mathrm{BL}}(X)=\Sigma_{j=0}^{\infty} \nu_{\alpha}^{j} u_{j}(X)$. To leading order $(j=0)$

$$
\frac{d}{d X}\left(\frac{u_{0}^{2}}{2}\right)=(-1)^{\alpha+1} \frac{d^{2 \alpha}}{d X^{2 \alpha}} u_{0}, \quad u_{0}( \pm \infty)=\mp 2 .
$$

For $\alpha=1$, we obtain the standard profile $u_{0}=$ $-2 \tanh X$. For $\alpha>1$, Eq. (2) cannot be solved analytically. However, for large $X$ the equation can be linearized because $u_{0}$ is close to its asymptotic constant value. For example, for large negative $X$, we set $u_{0}=2+w$, discard the quadratic term in $w$, and obtain, after integrating once, $(-1)^{\alpha+1} d^{2 \alpha-1} w / d X^{2 \alpha-1}=2 w$. This constant-coefficient ODE has solutions of the form $\mu \exp \left(\kappa_{\alpha} X\right)$, where $\mu$ is arbitrary and the "eigenvalue" $\kappa_{\alpha}$ is any of the $(2 \alpha-1)^{\text {th }}$ roots of $(-1)^{\alpha+1} 2$, i.e., for even $\alpha, \kappa_{\alpha}=2^{\beta} \exp (\imath(2 n+1) \beta \pi)$ and for odd $\alpha$, $\kappa_{\alpha}=2^{\beta} \exp (22 n \beta \pi)$, with $n=0,1, \ldots,(2 \alpha-2)$. Only the eigenvalues that have a positive real part are acceptable, because $w$ should vanish at $-\infty$. If all the modes with such eigenvalues are actually present then, for $X \rightarrow-\infty$, the solution to (2) tends to +2 in an oscillatory fashion and it is dominated by the mode $n_{\star}$ (and its complex conjugate), which has the smallest positive real part. In terms of the unstretched coordinates, this means that, in the neighborhood of the shock, the solution for even $\alpha$ displays damped oscillations with wavelength

$$
\left.\lambda_{\alpha}^{\text {th }}=2 \pi \nu_{\alpha}^{\beta}\left[2^{\beta} \sin \left[\left(2 n_{\star}+1\right) \beta \pi\right)\right]\right]^{-1}
$$

and with an e-folding rate

$$
\left.K_{\alpha}^{\mathrm{th}}=2^{\beta} \nu_{\alpha}^{-\beta} \cos \left[\left(2 n_{\star}+1\right) \beta \pi\right)\right] .
$$

The case of odd $\alpha$ is handled mutatis mutandis.

Such damped oscillations imply the presence of a pair of complex $k$ poles in wave-number space, whose signature, for real $k$, is a Lorentzian. This can be a bump or a trough, near wave number $2 \pi / \lambda_{\alpha}$, with width $\sim K_{\alpha}$ and amplitude $\sim K_{\alpha}^{-1}$. We present below a semi-numerical analysis to show that the solution of the DHB yields a bottleneck (bump).

The theory presented here can be applied to a wide class of problems ranging from the unforced, hyperviscous Burgers equation to the case of arbitrary, largescale, time-dependent or time-independent forcing as long as it does not modify the internal structure of shocks. Note also that such a linearized theory gives no prediction for the $\alpha$-dependence of the amplitude of the bottleneck. Furthermore, when more than one mode (or pair of complex-conjugate modes) with a positive real part is present (i.e., for $\alpha \geq 3$ ), linear theory does not tell us if the mode(s) with the smallest positive real part is (are) actually excited. Such issues require a global analysis of the boundary-layer equation (2) and not just of its large- $X$, linearized version. Except for the very standard case of ordinary dissipation, we do not know much about the properties of the solution to Eq. (2).
If we demand that $u_{0}(X=0)=0$ (which can always be achieved by a suitable translation), is the solution unique? By using a numerical, shooting method, we obtain evidence that, for $\alpha=2$, there is a unique solution that has $\left.u_{0}^{\prime}(X)\right|_{X=0}=-2.121530817618 \ldots$ and $\left.u_{0}^{\prime \prime}(X)\right|_{X=0}=0$. We can also obtain the value of this first derivative at the origin with $\simeq 10 \%$ accuracy by assuming that the solution has singularities on the imaginary axis at $X=Z_{\star}= \pm \mathrm{i} \Delta$ (a Painlevé-type argument indicates that, near such a singularity, to the leading order, $\left.u_{0}(X) \approx 120 /\left(X-Z_{\star}\right)\right)$. The vanishing of the second derivative implies that this unique solution is odd in the $X$ variable.

Direct numerical integration of the boundary-layer equation (2) is a greater challenge than the full DHB equation because, for the latter, we can take advantage of periodic boundary conditions. By using the value of $u_{0}^{\prime}(0)$, obtained by the shooting method discussed above, and $u_{0}^{\prime \prime}(0)=0$, we solve the third-order, boundary-layer equation for $\alpha=2$ numerically. We find $X_{C} \simeq 1.15$, the value of $X$ at which $u_{0}(X)$ first crosses the -2 asymptote. Next, we calculate $u_{0}^{\prime}\left(X_{C}\right)$ by using the Taylor expansion $u_{0}(X) \sim u_{0}^{\prime}(0) X+u_{0}^{\prime \prime \prime}(0) X^{3} / 3 !+u_{0}^{\prime \prime \prime \prime \prime}(0) X^{5} / 5 !+\ldots$ along with the known values of $u_{0}^{\prime}$ (from the shooting method), and $u_{0}^{\prime \prime \prime}(0)=2$ and $u_{0}^{\prime \prime \prime \prime \prime}(0)=-\left(u_{0}^{\prime}(0)\right)^{2}$ (from Eq. (21)). The linear theory suggests $u_{0}(X)=$ $-2+A e^{-K_{2}\left(X-X_{C}\right)} \sin 2 \pi\left(X-X_{C}\right) / \lambda_{2}$ for $X \geq X_{C}$; thence we obtain $u_{0}^{\prime}\left(X_{C}\right)$ in terms of $A$ and $\lambda_{2}$. By using the values of $u_{0}^{\prime}\left(X_{C}\right)$ (from the Taylor expansion above) and $\lambda_{2}$ (cf. Eq. (3)), we obtain $A \simeq-0.983$, which is within $1.7 \%$ of the value of $A(\simeq-0.966)$ that we get from the solution of the boundary-layer equation.

We now address the question of whether the Fourierspace manifestation of these oscillations is a bump or a trough. The Fourier transform $\gamma(k)$ of the real and even function $-u^{\prime}(X)$ is real and even [19]; and $\gamma(k)$ is the square root of the compensated energy spectrum. The rising of the compensated energy spectrum, in the intermediate regime, between the flat region near $k=0$ and the exponential decay at large $k$, is equivalent to $\gamma^{\prime \prime}(k)$ being positive; and $\left.\gamma^{\prime \prime}(k)\right|_{k \downarrow 0}=1 / 2 \pi \int_{0}^{\infty} d X X^{2} u^{\prime}(X)$. To solve for $\gamma^{\prime \prime}(k)$, we use $u^{\prime}(X)$ either from a numerical solution of Eq. (2) or from the linear theory above; we then perform a numerical integration over $X$; we obtain good agreement $(\simeq 9 \%)$ between the results of both these methods; and, indeed, we find that $\gamma^{\prime \prime}(k)$ is positive, so the spectrum has a bottleneck.

We turn now to a pseudospectral DNS of the DHB equation (11) with $\alpha=2,4,8$, and 16 , a $2 / 3$ rule for dealiasing, and a fourth-order, Runge-Kutta method for time-integration. The reference wavenumber $k_{r}=100$; the number of collocation points $N=2^{14}$, the time step $\delta t=10^{-4}$, and the hyperviscosity coefficients are $\nu_{2}=5 \times 10^{-3}, \nu_{4}=5 \times 10^{-8}, \nu_{8}=5 \times 10^{-14}$, and $\nu_{16}=10^{-20}$.

The steady-state, compensated energy spectra $E_{k}^{c} \equiv$ 

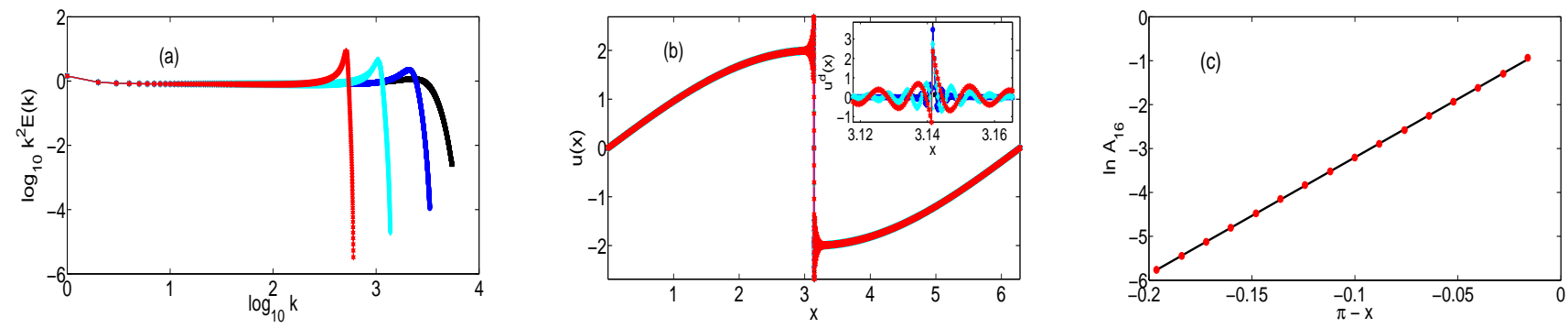

FIG. 1. (Color online) Plots for the DHB Eq. (1): (a) Log-log plots of the compensated energy spectrum $k^{2} E(k)$ versus $k$ for $\alpha=2$ (black squares), $\alpha=4$ (blue filled-circles), $\alpha=8$ (cyan diamonds), and $\alpha=16$ (red hexagons). (b) Plots of the steady-state solution $u(x)$ for the same values of $\alpha$ as in (a); these are indistinguishable from $u^{o}(x)$ (thick magenta line) away from $x=\pi$; inset: plots of $u^{d}(x)$ versus $x$ around $x=\pi$. (c) Semilog plot of $A_{16}$ (red filled circles) versus $\pi-x$; the black line is the fit.

$k^{2} E(k)$ (Fig. (1a)) show clear bottlenecks; the height of the bottleneck peak increases with $\alpha$; but $k_{b}^{\alpha}$, the wavenumber of this peak, decreases as we increase $\alpha$. We now investigate the real-space manifestation of this bottleneck. In Fig. (1b) we plot the steady-state solution of the DHB equation; this shows that our numerical solution agrees with the outer solution $u^{o}(x)=$ $2 \operatorname{sgn}(\pi-x) \sin (x / 2)$ away from the shock. However, in a thin boundary layer around the shock at $x=\pi$, there are conspicuous oscillations that become prominent when we plot the difference between the solution of the DHB equation and the outer solution, $u^{d}(x) \equiv$ $u(x)-u^{o}(x)$, versus $x$ [Fig. (1b), inset]. The characteristic wavelength of these oscillations is $\lambda_{\alpha}$; for the representative case $\alpha=16$, we find $\lambda_{16}=0.0122$. Similarly, from plots of the compensated spectra [Fig. (1a)] we obtain $\lambda_{16}=0.0121$. Furthermore, the theoretical prediction for the wavelength of these oscillations (cf. Eq.(3i) ) yields $\lambda_{16}^{\text {th }}=0.0120$. The bottleneck has a finite width because of the decaying envelope of the oscillations in real space as we move away from the shock (cf. Eq.(44)). We obtain the amplitude $A_{\alpha}$ of these oscillations [Fig. (1b)] as a function of $(x-\pi)$ and find, numerically, that $A_{\alpha} \sim \exp \left[K_{\alpha}(x-\pi)\right]$ as shown in Fig. (1c). For $\alpha=16$ we obtain, from our DNS, an e-folding rate $K_{16} \simeq 26.61$, whereas our theoretical prediction in Eq.(4) yields $K_{16}^{\text {th }} \simeq 26.54$. Thus, we find excellent agreement between our theoretical predictions (Eqs.(3) and (4)) and our numerical results for both the wavelength of the oscillations and the e-folding rate.

Our results carry over to the stochastically forced hyperviscous Burgers equation (SHB) and to the 3D hyperviscous Navier-Stokes (HNS) equations, both of which exhibit turbulence. Let us first examine bottlenecks in the SHB equation [14], with a white-in-time, Gaussian random force with zero mean, an ultraviolet cutoff at $N / 8$, and a spectrum $\sim k^{-1}$. The velocity field for the SHB shows shocks at various length scales; and the resulting energy spectrum shows an inertial-range scaling $E(k) \sim k^{-5 / 3}$. In Fig. (2a) we give a representative plot of the compensated energy spectrum $k^{5 / 3} E(k)$, for $\alpha=8$; this shows such an inertial range followed by a prominent bottleneck that peaks at a wavenumber $k_{b}^{8} \simeq 890$. We measure the correlation function $\langle u(x) u(x+l)\rangle$, averaged over statistically independent configurations, which show oscillations [Fig. (2a), inset]; these are the realspace manifestations of this bottleneck. The wavelength of these oscillations is $\simeq 0.00706$; and the corresponding wavenumber is $\simeq 889.97$, in agreement with the wavenumber at which the bottleneck shows a peak in Fig. (2a).

The 3D hyperviscous Navier-Stokes (HNS) equation for an incompressible velocity field $\mathbf{u}(\mathbf{x}, t)$ is

$$
\begin{aligned}
\frac{\partial \mathbf{u}}{\partial t}+\mathbf{u} \cdot \nabla \mathbf{u}(\mathbf{x}, t) & =-\nabla p-\nu_{\alpha}\left(-\nabla^{2}\right)^{\alpha} \mathbf{u}(\mathbf{x}, t)+\mathbf{f}(\mathbf{x}, t) \\
\nabla \cdot \mathbf{u} & =0 .
\end{aligned}
$$

We integrate it by a pseudospectral method with a $2 / 3$ dealiasing rule, an Adams-Bashforth scheme for time marching, $512^{3}$ collocation points, $\alpha=4$, and $\nu_{4}=$ $10^{-14}$. We force the 3D HNS equation to a statistically steady state by using the constant-energy-injection method described in Ref. [15]. In Fig. (2b), we show a representative plot of the compensated energy spectrum $E^{c}(k) \equiv k^{5 / 3} E(k)$; this shows a bottleneck between the inertial and dissipation ranges. The correlation function $D(l)=\langle\mathbf{u}(\mathbf{x}) \cdot \mathbf{u}(\mathbf{x}+\mathbf{l})\rangle$, averaged over five configurations that are separated from each other by $6 \tau_{I}$, where $\tau_{I}$ is the integral-scale eddy turnover time, shows gentle oscillations [Fig. (2c)], which are the real-space manifestations of this bottleneck. These oscillations can be seen clearly in $D^{o}(l)$ [Fig. (2c), inset], which is obtained by subtracting the linear, decaying trend from $D(l)$. The wavelength of these oscillations is $\simeq 0.1665$ and the corresponding wavenumber is $\simeq 37.7$, in agreement with the wavenumber at which the bottleneck shows a peak in Fig. (2b).

We have provided a theoretical explanation for energyspectra bottlenecks in the DHB equation by combining analytical and numerical studies. These bottle- 

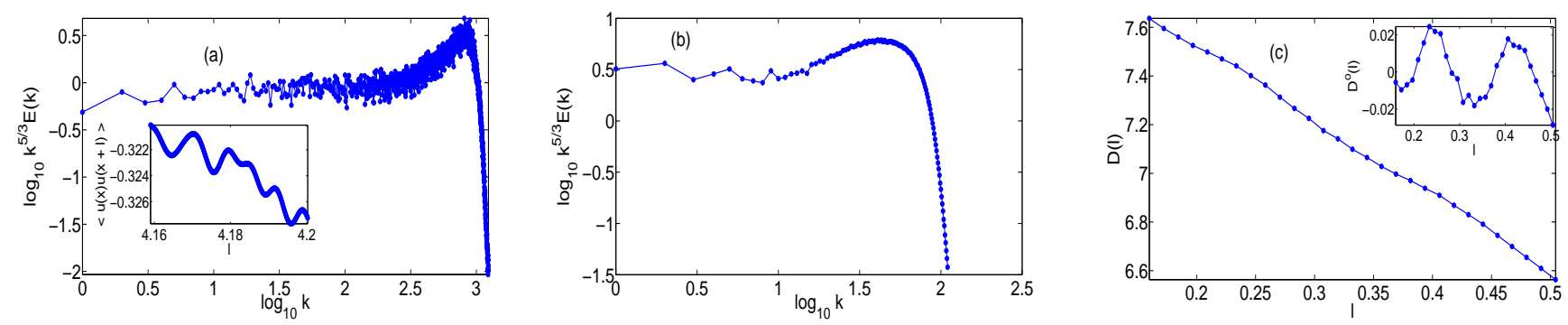

FIG. 2. (Color online) (a) Log-log plot of the compensated energy spectrum $k^{5 / 3} E(k)$ versus $k$ for the stochastically forced hyperviscous Burgers (SHB) equation with $\alpha=8$ and (inset) a plot of its correlation function $\langle u(x) u(x+l)\rangle$ showing oscillations of wavelength $\lambda_{8}^{\mathrm{SHB}}$, which is inversely related to the wavenumber of the bottleneck in the energy spectrum. (b) The compensated energy spectrum $k^{5 / 3} E(k)$ for the 3D HNS equation $(\alpha=4)$ with a bottleneck peak at wavenumber $K_{b, \alpha}^{\mathrm{HNS}}=40$. (c) A plot of the correlation function $D(l)$ versus $l$ for the 3D HNS equation; inset: oscillations in a plot of the function $D^{o}(l)$, which we obtain by subtracting the linear, decaying trend from $D(l)$.

necks appear as a natural consequence of oscillations in the velocity profiles in the vicinity of a shock. Earlier studies [16, 17] have seen such oscillations in the DHB case but have not associated them with bottlenecks in energy spectra. Furthermore, we have shown that energy-spectra bottlenecks in the SHB and the 3D HNS equations, which exhibit turbulence, are associated with damped oscillations in real-space velocity correlation functions. This association has not been made hitherto, even though there have been attempts to explore real-space manifestations of bottlenecks in energy spectra [10]. Our work confirms that the larger the dissipativity $\alpha$, the more pronounced is the bottleneck [7, 11, 18]. Thus, by increasing $\alpha$, we have uncovered clearly the real-space manifestations of these bottlenecks. Energy spectra for homogeneous isotropic turbulence in the 3D NS equation $(\alpha=1)$ show a mild bottleneck [9, 11]; we expect, therefore, that there should be weak oscillations in real-space velocity correlation functions. The detection of such weak oscillations is an important challenge for experiments and DNS.

We thank W. Pauls, K.R. Sreenivasan, and A. Wirth for discussions, CSIR, UGC, and DST (India) for support, and SERC (IISc) for computational resources. UF and RP are members of the International Collaboration for Turbulence Research; UF, RP, and SSR acknowledge support from the COST Action MP0806; UF and SSR's work was supported by ANR "OTARIE" BLAN072_183172; DB and RP thank the Observatoire de la Côte d'Azur for hospitality; and UF thanks the Indian Institute of Science for the DST-IISc Centenary Professorship.

* Also at Jawaharlal Nehru Centre For Advanced Scientific Research, Jakkur, Bangalore, India

[1] A.N. Kolmogorov, Dokl. Akad. Nauk. SSSR 30, 299303 (1941).

[2] U. Frisch, Turbulence: the Legacy of A.N. Kolmogorov,
(Cambridge University Press, Cambridge, UK, 1995).

[3] J. von Neumann, Recent theories of turbulence (1949). In Collected works (1949-1963) 6, 37-472, ed. A.H. Taub. Pergamon Press, New York, 1963; as first observed by von Neumann, this follows from the (conjectured) analyticity of the solution of the Navier-Stokes equation.

[4] G. Falkovich, Phys. Fluids, 6, 1411 (1994).

[5] D. Lohse and A. Müller-Groelling, Phys. Rev. Lett., 74, 1747 (1995).

[6] H.K. Pak, W.I. Goldburg, A. Sirivat, Fluid Dynamics Research, 8, 19 (1991); Z.-S. She and E. Jackson, Phys. Fluids A, 5, 1526 (1993); S.G. Saddoughi and S.V. Veeravalli, J. Fluid Mech., 268, 333 (1994).

[7] W. Dobler, N.E.L. Haugen, T.A. Yousef and A. Brandenburg, Phys. Rev. E, 68, 026304 (2003).

[8] Z.-S. She, G. Doolen, R.H. Kraichnan, and S.A. Orszag, Phys. Rev. Lett., 70, 3251 (1993); P.K. Yeung and Y. Zhou, Phys. Rev. E, 56, 1746 (1997); T. Gotoh, D. Fukayama, and T. Nakano, Phys. Fluids, 14, 1065 (2002); M.K. Verma and D.A. Donzis, J. Phys. A: Math. Theor., 40, 4401 (2007); P.D. Mininni, A. Alexakis, and A. Pouquet, Phys. Rev. E, 77, 036306 (2008).

[9] Y. Kaneda, et al., Phys. Fluids, 15, L21 (2003); T. Isihara, T. Gotoh, and Y. Kaneda Annu. Rev. Fluid Mech., 41, 165 (2009); S. Kurien, M.A. Taylor, and T. Matsumoto, Phys. Rev. E, 69, 066313 (2004).

[10] D.A. Donzis and K.R. Sreenivasan J. Fluid Mech., 657, 171 (2010); DOI: 10.1017/S0022112010001400, suggest that bottlenecks might go away as the Reynolds number goes to infinity.

[11] U. Frisch, et al., Phys. Rev. Lett., 101, 144501 (2008).

[12] J. Bec and K. Khanin, Phys. Rep., 447, 1 - 66 (2007).

[13] See, e.g., J.-P. Hansen and I.R. McDonald, Theory of Simple Liquids, (Academic Press, London, 2006); J.L. Yarnell, M.J. Katz, R.G. Wenzel, and S.H. Koenig, Phys. Rev. A, 7, 2130 (1973).

[14] D. Mitra, J. Bec, R. Pandit, and U. Frisch, Phys. Rev. Lett., 94, 194501 (2005).

[15] A.G. Lamorgese, D.A. Caughey, and S.B. Pope, Phys. Fluids, 17, 015106 (2005).

[16] J.P. Boyd, J. Atmos. Sci., 49, 128 (1992); J. Sci. Comput., 9, 81 (1994).

[17] W. Pauls and J. Zudrop, unpublished. 
[18] D. Biskamp, E. Schwarz, and A. Celani, Phys. Rev. Lett., 81, 4855 (1998); D. Biskamp and W.-C. Müller, Phys. Plasmas, 7, 4889 (2000).

[19] This can be checked numerically. Also, because $-u^{\prime}(0)>$ 0 and we are within the radius of convergence of the Tay- lor expansion of $u(X)$, as obtained from the Painlevétype analysis, $\gamma(k)$ cannot suddenly become negative without being positive for at least some small region. 